\title{
Novel imaging system for positioning of the indocyanine green (ICG) target; visible projection of the near-infrared fluorescence image
}

\author{
In Hee Shin ${ }^{1}$, Seok Ki Kim², Joo Beom Eom ${ }^{1}$, Jae Seok Park ${ }^{1}$, Hyeong Ju Park ${ }^{1}$, In-Kyu Park ${ }^{3}$, \\ Byeong-Il Lee ${ }^{1^{*}}$ \\ ${ }^{1}$ Medical Photonics Research Center, Korea Photonics Technology Institute, Gwangju, South Korea \\ ${ }^{2}$ Department of Nuclear Medicine, National Cancer Center, Goyang, South Korea \\ ${ }^{3}$ Department of Biomedical Sciences, Chonnam National University Medical School, Gwangju, South Korea \\ Email: ihshin@kopti.re.kr, skkim@ncc.re.kr, jbeom@kopti.re.kr, jspark@kopti.re.kr, hj202@kopti.re.kr, pik96@chonnam.ac.kr, \\ *bilee@kopti.re.kr
}

Received 17 July 2013; revised 21 August 2013; accepted 5 September 2013

Copyright (C) 2013 In Hee Shin et al. This is an open access article distributed under the Creative Commons Attribution License, which permits unrestricted use, distribution, and reproduction in any medium, provided the original work is properly cited.

\begin{abstract}
Background: Even though NIR fluorescence imaging has many advantages in SLN mapping and cancer detection, NIR fluorescence imaging shows a serious drawback that NIR cannot be detected by the naked eye without any detectors. This limitation further disturbs accurate SLN detection and adequate tumor resection resulting in the presence of cancerous cells near the boundaries of surgically removed tissues. Materials and methods: To overcome the drawback of the conventional NIR imaging method, we suggest a novel NIR imaging system which can make the NIR fluorescence image visible to the naked eye as NIR fluorescence image detected by a video camera is processed by a computer and then projected back onto the NIR fluorescence excitation position with a projector using conspicuous color light. Image processing techniques were used for projection onto the exact position of the NIR fluorescence image. Also, we implemented a phantom experiment to evaluate the performance of the developed NIR fluorescence projection system by use of the ICG. Results: The developed NIR fluorescence projection system was applied in normal mouse model to confirm the usefulness of the system in the clinical field. A BALB/c nude mouse was prepared to be applied in normal mouse model and $0.25 \mathrm{mg} / \mathrm{ml}$ stock solution of the ICG was injected through a tail vein of the mouse. From the application in normal mouse model, we could confirm that the injected ICG stayed in the liver of the mouse and verify that the projection system projected the ICG

\footnotetext{
${ }^{*}$ Corresponding author.
}

fluorescence image at the exact location of the ICG by performing laparotomy of the mouse. Conclusions: From the application in normal mouse model, we could verify that the ICG fluorescence image was precisely projected back on the site where ICG fluorescence generated. It can be demonstrated that the NIR fluorescence projection system can make it possible to visualize the invisible NIR fluorescence image and to realize that SLN mapping and cancer detection in clinical surgery.

Keywords: Fluorescence Imaging; NIR Fluorescence; Optical Projection; Near-Infrared Fluorescence; ICG

\section{BACKGROUND}

Currently, near-infrared (NIR) fluorescence imaging has been regarded as one of the most important experimental techniques that can be used to visualize cancer cells during surgery because surgeons rely on palpation and visual inspection in surgical practices. Actually, use of NIR fluorescence imaging was demonstrated in patients with glioma and liver cancer [1-4]. Advantages of NIR fluorescence light $(700-900 \mathrm{~nm})$ include high tissue penetration (up to several centimeters deep) and low autofluorescence providing sufficient signal to background ratio [5]. Moreover, as the human eye is insensitive to NIR wavelengths, the use of NIR light does not interfere with the surgical field. Recent preclinical and clinical data have demonstrated that NIR fluorescence imaging using the NIR fluorescence agent indocyanine green (ICG) enables real-time transcutaneous and intraoperative visualization of lymphatic channels and detection of 
the sentinel lymph node (SLN) [6-14]. Especially SLN mapping, as introduced for the management of breast cancer by Giuliano et al. is currently regarded as the standard of care for staging of the axilla $[4,15,16]$. Therefore, NIR fluorescence imaging can be applied as an alternative for conventional techniques used for SLN mapping.

However, even though NIR fluorescence imaging has many advantages in SLN mapping and cancer detection, NIR fluorescence imaging shows a serious drawback that NIR cannot be detected by the naked eye without any detectors. This limitation further disturbs accurate SLN detection and adequate tumor resection resulting in the presence of cancerous cells near the boundaries of surgically removed tissues. In surgical practice, surgeons should use extra-instruments such as the special goggles to accurately detect NIR fluorescence [17-19]. Use of extra-instruments, however, may cause a great deal of inconvenience for surgeons on operations.

In this paper, we suggest a NIR imaging system that can make the NIR fluorescence image visible to the naked eye as the NIR fluorescence image detected by a video camera is processed by a computer and then projected back onto the NIR fluorescence excitation position with a projector using conspicuous color light (Figure 1).

\section{MATERIALS AND METHODS}

\subsection{Composition of a NIR Fluorescence Projection System}

The NIR fluorescence image's projection system and its operation principle are shown in Figures 1 and 2. The projection system consists of a CCD camera (Guppy, PRO F-031B) for acquisition of the ICG fluorescence image, a machine vision macro lens (VS Technology, VS-MC35) combined with a band-pass filter (Thorlabs, FB830-10, center wavelength: $830 \mathrm{~nm}$ ), and a projector

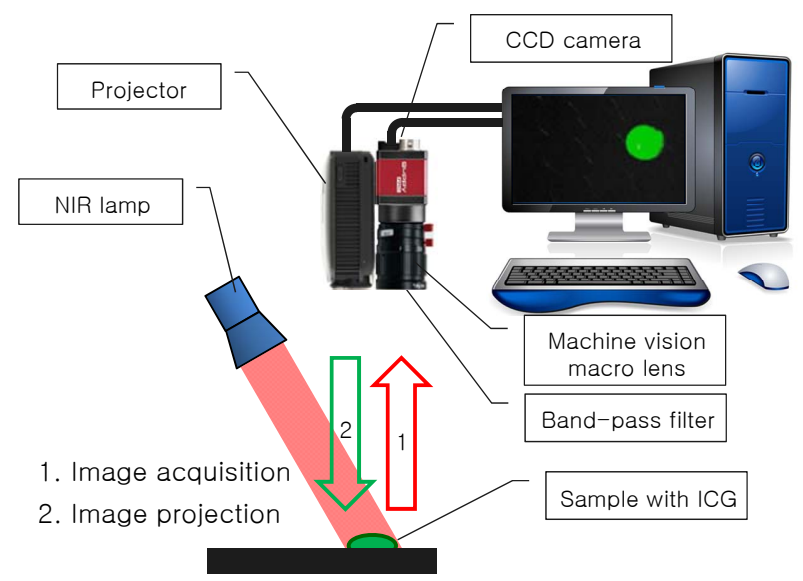

Figure 1. Schematic diagram of the NIR fluorescence image's projection system.

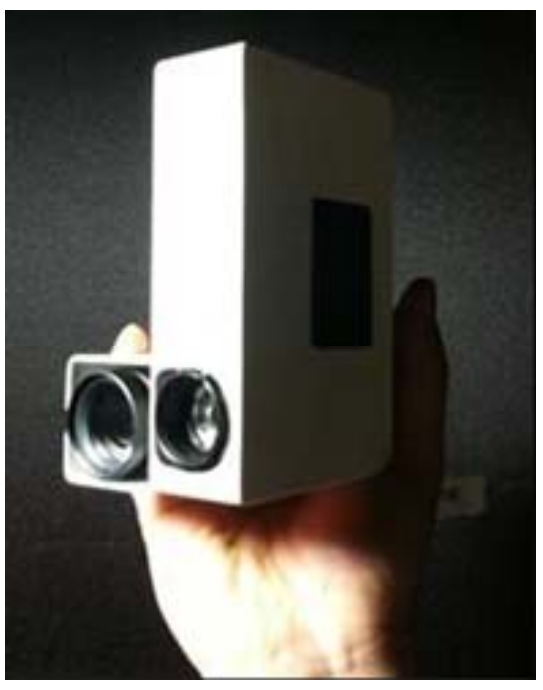

Figure 2. The picture of the NIR fluorescence image's projection system.

(Optoma, PK320) as shown in Figure 1. The machine vision macro lens is put in the CCD camera and the projector is also combined with the CCD camera as a probe for the NIR fluorescence acquisition and projection.

As a NIR optical source, light emitting diodes (LEDs) which have $760 \mathrm{~nm}$ of center wavelength are used for excitation of ICG fluorescence. The ICG fluorescence image excited by the NIR lamp is acquired by the CCD camera and then the fluorescence image is processed by a computer. In the process of the image processing, the fluorescence image has conspicuous color for emphasis of the projected image and has adjusted image size and location for accordance between the acquired image and the projected image. Finally, the processed ICG fluorescence image is accurately projected back onto the subject and users of the projection system can confirm the exact shape and location of the ICG fluorescence image to the naked eye.

\subsection{Image Processing for NIR Fluorescence Projection}

A square image sample $(1 \mathrm{~cm} \times 1 \mathrm{~cm})$ was prepared to confirm the image acquisition and projection as shown in Figure 3(a) and the state of the acquired image by the CCD camera and the projected image by the projector were checked according to the height of the projection system. For the experiment, the band-pass filter was temporarily removed from the CCD camera-projector probe. Figure 4 shows the size variation of the square image sample according to height of the projection system. When the height of the projection system increased as $21,23,25,27$, and $30 \mathrm{~cm}$ from the bottom, the size of the images acquired by the CCD camera decreased linearly as $7.8,7.35,6.8,6.35$, and $5.7 \mathrm{~cm}$ without the length 


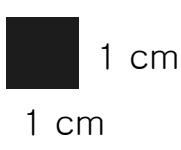

(a)

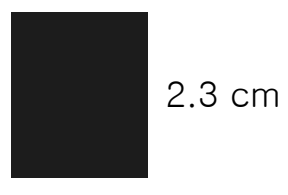

$1.9 \mathrm{~cm}$

(b)

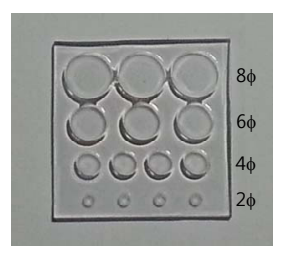

(a)

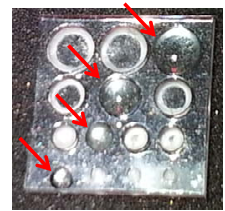

(b)

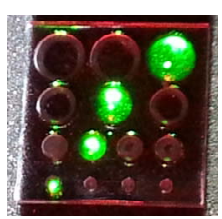

(c)
Figure 3. The square image sample (a) and the projected image of the sample (b).

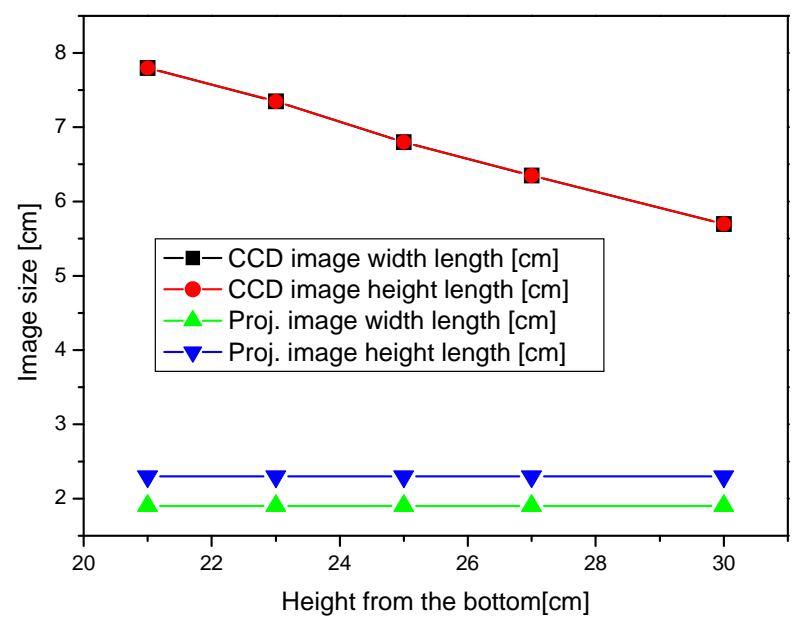

Figure 4. Size variation of the square image sample according to height of the CCD camera-projector probe.

difference between the width and the height. However, the size of the projected images was uniform as $1.9 \mathrm{~cm}$ of width length and $2.3 \mathrm{~cm}$ of height length although the height of the projection system increased. Therefore, we corrected the length difference between the width and the height and the size difference between the real image (1 $\mathrm{cm} \times 1 \mathrm{~cm})$ and the projected image $(1.9 \mathrm{~cm} \times 2.3 \mathrm{~cm}$, Figure 3(b)) by using the image processing technique. The camera used to acquire the fluorescence image is a CCD camera with 14 bit greyscale. Therefore, the NIR fluorescence in the acquired image provides just a white color and the white color decreases visibility when the fluorescence image is projected back onto the subject. So, conspicuous color which can be controlled depending on the surface color of the subject was merged in the region of the NIR fluorescence. The image processing was implemented by using the Labview (National Instrument, USA).

\subsection{Performance Evaluation of the NIR Fluorescence Projection System}

To evaluate performance of the NIR fluorescence projection system, a phantom which has circular holes 2, 4, 6, and $8 \mathrm{~mm}$ wide and $1 \mathrm{~mm}$ deep was manufactured as shown in Figure 5(a). ICG (25-mg vials) was purchased from Daiichi Sankyo Propharma Co. (Osaka, Japan) and
Figure 5. (a) The phantom to evaluate performance of the NIR fluorescence projection system; (b) the resuspended ICG in the phantom (red arrows show the exact locations of the ICG); (c) the projection image of the ICG fluorescence by the NIR fluorescence projection system.

was resuspended in 10cc of sterile water for injection to yield a $1.25 \mu \mathrm{g} / \mathrm{ml}$ stock solution. The resuspended ICG was put into the circular holes of the phantom and the fluorescence image of the ICG in the phantom was acquired and projected by the projection system as shown in Figures 5(b) and (c). From the fluorescence image of the ICG in the phantom, we could confirm that the projected fluorescence image coincides with the expressed fluorescence image accurately and the projection system is capable of projecting the small fluorescence image of $2 \mathrm{~mm}$ size.

\section{RESULTS}

\section{Application in Normal Mouse Model of the NIR Fluorescence Projection System}

The NIR fluorescence projection system was applied in normal mouse model to confirm the usefulness of the system in clinical field.

A BALB/c nude mouse was prepared to be applied in normal mouse model and $0.25 \mathrm{mg} / \mathrm{ml}$ stock solution of the ICG was injected through a tail vein of the mouse. Acquisition and projection of the fluorescence image were implemented by the NIR fluorescence projection system after ten seconds of the tail vein injection. Figures 6(a) and (b) show the ICG fluorescence images acquired and projected by the projection system on the skin of the mouse, respectively. We could confirm that the injected ICG stayed in the liver of the mouse from the Figures 6(a) and (b) and verify that the projection system projected the ICG fluorescence image at the exact position of the fluorescence expression by performing laparotomy of the mouse as shown in the Figures 6(c).

From the application in normal mouse model of the NIR fluorescence projection system, we can demonstrate that newly developed projection system can be a novel tool for visualization and positioning of the NIR fluorescence.

\section{CONCLUSIONS}

In summary, we have developed a novel NIR fluores- 

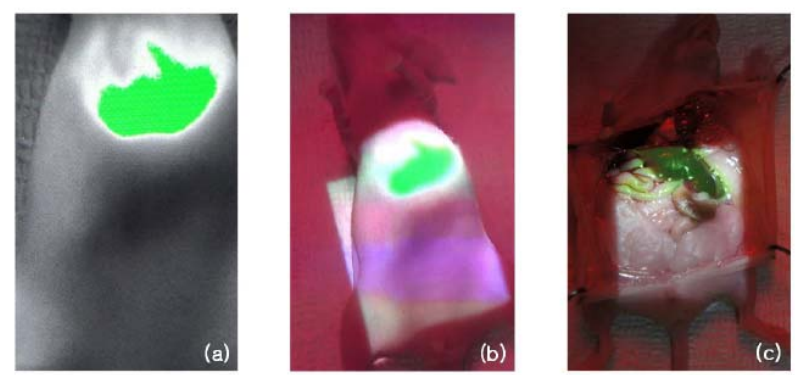

Figure 6. (a) The ICG fluorescence image by the CCD camera; (b) projection of the ICG fluorescence image and (c) the real projection image of the ICG fluorescence at the liver of the nude mouse.

cence projection system for visualization and positioning of the NIR fluorescence. The operation principle for the projection system is simple in that the ICG fluorescence image excited by the NIR lamp is acquired by the CCD camera and then the fluorescence image is processed by a computer. In the process of the image processing, the fluorescence image has a conspicuous color for emphasis of the projected image and has adjusted image size and location for accordance between the acquired image and the projected image. Finally, the processed ICG fluorescence image is precisely projected back onto the subject.

By use of the NIR fluorescence projection system and the normal mouse model, we observed the visualization and positioning of the NIR fluorescence projection image. From the experiment by the normal mouse model, it can be demonstrated that the NIR fluorescence projection system can make it possible to visualize and position the invisible NIR fluorescence image.

Also, we can verify that the NIR fluorescence projection system can make it possible to realize the SLN mapping and cancer detection in clinical surgery.

\section{ACKNOWLEDGEMENTS}

This work was supported by the Bio \& Medical Technology Development Program of the National Research Foundation funded by the Korean government (MEST) (2011-0019794).

\section{REFERENCES}

[1] Stepp, H., Beck, T., Pongratz, T., Meinel, T. Tonn, Ch. J., Kreth, F.W. and Stummer, W. (2007) ALA and malignant glioma: Fluorescence-guided resection and photodynamic treatment. Journal of Environmental Pathology, Toxicology and Oncology, 26, 157-164. doi:10.1615/JEnvironPatholToxicolOncol.v26.i2.110

[2] Ishizawa, T., Fukushima, N., Shibahara, J., Mauda, K., Tamura, S., Aoki, T., Hasegawa, K., Beck, Y., Fukayama, M. and Kokubo, N. (2009) Real-time identification of liver cancers by using Indocyanine green fluorescent imaging. Cancer, 115, 2491-2504. doi:10.1002/cncr.24291
[3] Nguyen, N.Q., Biankin, A.V., Leong, R.W., Chang, D.K., Cosman, P.H., Delaney, P., Kench, J.G. and Merrett, N.D. (2009) Real time intraoperative confocal laser microscopy guided surgery. Annals of Surgery, 249, 735-737. doi:10.1097/SLA.0b013e3181a38f11

[4] Miego, J.S.D., Hutteman, M., van der Vorst, J.R., Kuppen, P.J.K., Que, I., Dijkstra, J., Kaijzel, E.L., Prins, F., Lowik, C.W.G.M., Smit, V.T.H.B.M., van de Velde, C.J.H. and Vahrmeijer, A.L. (2011) Image-guided tumor resection using real-time near-infrared fluorescence in a syngeneic rat model of primary breast cancer. Breast Cancer Research and Treatment, 128, 279-689.

[5] Frangioni, J.V. (2008) New technologies for human cancer imaging. Journal of Clinical Oncology, 26, 40124021. doi:10.1200/JCO.2007.14.3065

[6] Hirche, C., Murawa, D., Mohr, Z., Kneif, S. and Hunerbein, M. (2010) ICG fluorescence-guided sentinel node biopsy for axillary nodal staging in breast cancer. Breast Cancer Research and Treatment, 121, 373-378. doi:10.1007/s10549-010-0760-z

[7] Hojo, T., Nagao, T., Kikuyama, M., Akashi, S. and Kinoshita, T. (2010) Evaluation of sentinel node biopsy by combined fluorescent and dye method and lymph flow for breast cancer. Breast, 19, 210-213. doi:10.1016/j.breast.2010.01.014

[8] Kitai, T., Inomoto, T., Miwa, M. and Shikayama, T. (2005) Fluorescence navigation with indocyanine green for detecting sentinel lymph nodes in breast cancer. Breast Cancer, 12, 211-215. doi:10.2325/jbcs.12.211

[9] Murawa, D., Hirche, C., Dresel, S. and Hunerbein, M. (2009) Sentinel lymph node biopsy in breast cancer guided by indocyanine green fluorescence. British Journal of Surgery, 96, 1289-1294.

doi:10.1002/bjs.6721

[10] Sevick-Muraca, E.M., Sharma, R., Rasmussen, J.C., Marshall, M.V., Wendt, J.A., Pham, H.Q., Bonefas, E., Houston, J.P., Sampath, L., Adams, K.E., Blanchard, D.K., Fisher, R.E., Chiang, S.B., Elledge, R. and Mawad, M.E. (2008) Imaging of lymph flow in breast cancer patients after microdose administration of a near-infrared fluorophore: Feasibility study. Radiology, 246, 734-741. doi:10.1148/radiol.2463070962

[11] Tagaya, N., Yamazaki, R., Nakagawa, A., Abe, A., Hamada, K., Kubota, K. and Oyama, T. (2008) Intraoperative identification of sentinel lymph nodes by near-infrared fluorescence imaging in patients with breast cancer. American Journal of Surgery, 195, 850-853. doi:10.1016/j.amjsurg.2007.02.032

[12] Tanaka, E., Chen, F.Y., Flaumenhaft, R., Graham, G.J., Laurence, R.G. and Frangioni, J.V. (2009) Real-time assessment of cardiac perfusion, coronary angiography, and acute intravascular thrombi using dual-channel near-infrared fluorescence imaging. Journal of Thoracic and Cardiovascular Surgery, 138, 133-140. doi:10.1016/j.jtcvs.2008.09.082 PMid:19577070 PMCid:2706783

[13] Troyan, S.L., Kianzad, V. and Gibbs-Strauss, S.L. (2009) The FLARE intraoperative near-infrared fluorescence 
imaging system: a first-in-human clinical trial in breast cancer sentinel lymph node mapping. Annals of Surgical Oncology, 16, 2943-2952. doi:10.1245/s10434-009-0594-2

[14] Miego, J.S.D., Troyan, S.L., Hutteman, M., Donohue, K.J., van der Vorst, J.R., Stockdale, A., Liefers, G.J., Choi, H.S., Gibbs-Strauss, S.L., Putter, H., Gioux, S., Kuppen, P.J.K., Ashitate, Y., Lowik, C.W.G.M., Smit, V.T.H.B.M., Oketokoun, R., Ngo, L.H., van de Velde, C.J.H., Frangioni, J.V. and Vahrmeijer, A.L. (2011) Towards optimization of imaging system and lymphatic tracer for near-infrared fluorescent sentinel lymph node mapping in breast cancer. Annals of Surgical Oncology, 18, 2483-2491. doi:10.1245/s10434-011-1566-X

[15] Giuliano, A.E., Kirgan, D.M., Geunther, J.M. and Morton, D.L. (1994) Lymapathic mapping and sentinel lymphadenectomy for breast cancer. Annals of Surgery, 220, 391-398. doi:10.1097/00000658-199409000-00015

[16] Cox, C.E., Pendas, S., Cox, J.M., Joseph, E., Shons, A.R., Yeatman, T., Ku, N.N., Lyman, G.H., Berman, C., Haddad, F. and Reintgen, D.S. (1998) Guidelines for sentinel node biopsy and lymphatic mapping of patients with breast cancer. Annals of Surgery, 227, 645-651. doi:10.1097/00000658-199805000-00005

[17] Liu, Y., Bauer, A.Q., Akers, W., Sudlow, G., Liang, K., Shen, D., Berezin, M., Culver, J.P. and Achilefu, S. (2011) Hands-free, wireless goggles for near-infrared fluorescence and real-time image-guided surgery. Journal of Surgery, 149, 689-698. doi:10.1016/j.surg.2011.02.007

[18] Collins, L., Schnitt, S., Achacoso, N., Fletcher, S., Nekhlyudov, L., Haque, R., Quesenberry, C. and Habel, L. (2009) Outcome of women with ductal carcinoma in situ treated with breast-conserving surgery alone: A case-control study of 225 patients from the cancer research network. Modern Pathology, 22, 34A-35A.

[19] Vicini, F.A., Kestin, L.L., Goldstein, N.S., Chen, P.Y., Pettinga, J., Frazier, R.C. and Martinez, A.A. (2000) Impact of young age on outcome in patients with ductal carci-noma in-situ treated with breast-conserving therapy. Journal of Clinical Oncology, 18, 296-306. 